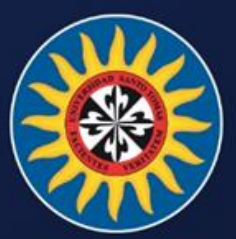

UNIVERSIDAD SANT0 TOMAS PRIMER CLAUSTRO UNIVERSITARIO DE COLOMBIA

VIGILADA MINEDUCACIÓN - SNIES 1704 


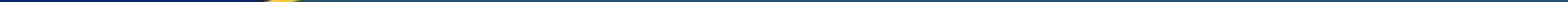




\section{Memorias del evento: Reflexiones Pedagógicas en los contextos educativos desde las competencias ciudadanas en la sociedad}
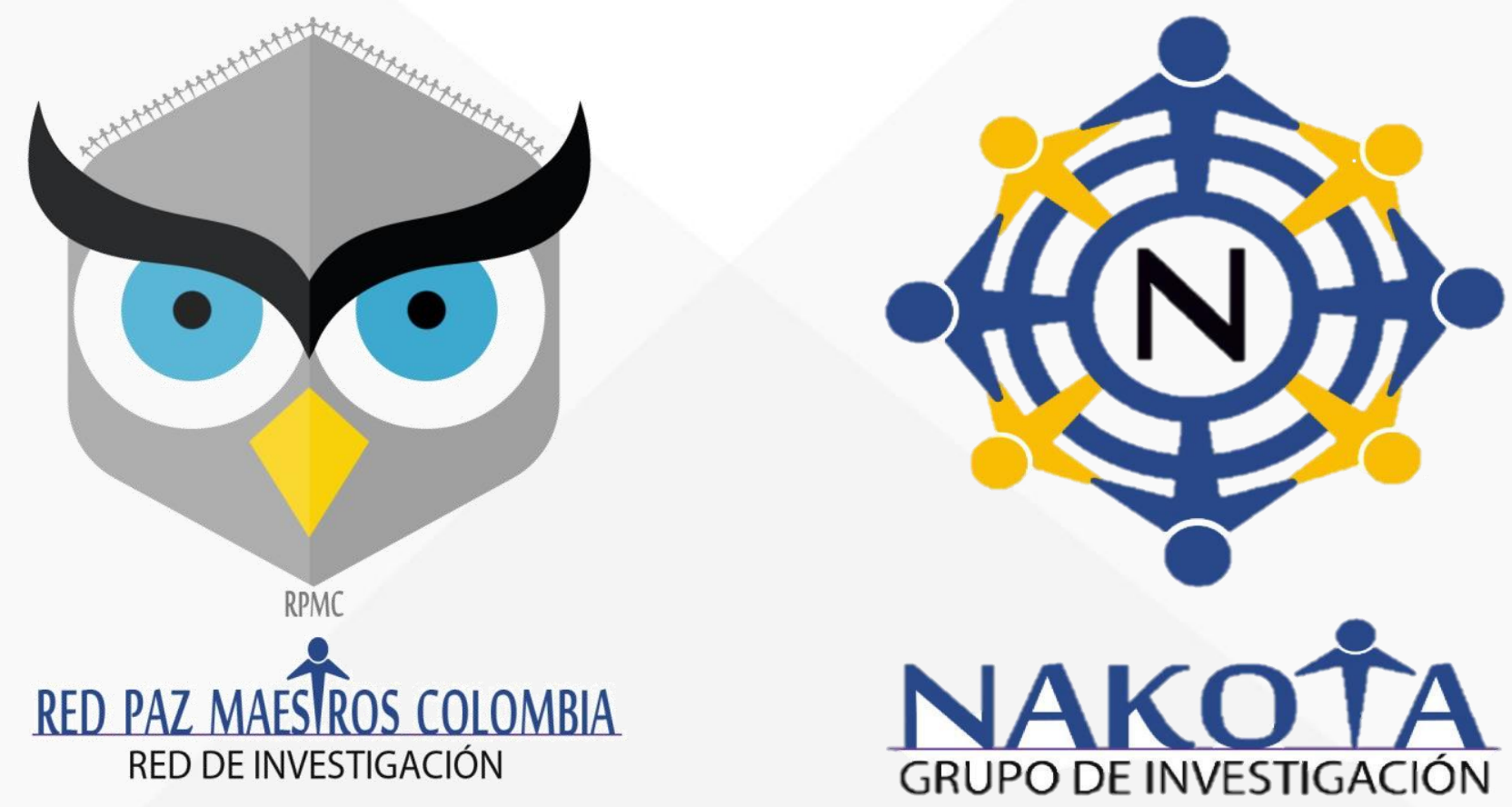


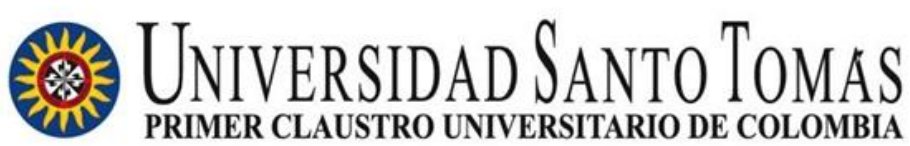 \\ VIGILADA MINEDUCACIÓN - SNIES 1704

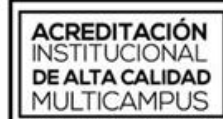 \\ MULTICAMPUS \\ Universidad Santo Tomás}

Centro de Atención Universitaria CAU Villavicencio

Memorias del evento:

Reflexiones Pedagógicas en los contextos educativos desde las competencias ciudadanas en la sociedad

Red Paz Maestros Colombia

Grupo de Investigación Nakota.

DUAD 


\section{Autores:}

\begin{tabular}{|c|c|c|c|}
\hline NOMBRE & CvLac & Google Scholar & ORCID \\
\hline $\begin{array}{l}\text { Daniel Alejandro } \\
\text { Contreras Castro }\end{array}$ & $\begin{array}{l}\text { https://scienti.minciencias.gov.co/cvlac/visualizad } \\
\underline{\text { or/generarCurriculoCv.do?cod } r h=0000031500}\end{array}$ & $\begin{array}{l}\text { https://scholar.google.es/citations?user=1yG } \\
\underline{\text { Vzq0AAAAJ\&hl=es\&oi=ao }}\end{array}$ & $\begin{array}{l}\underline{\text { https://orcid.org/00 }} \\
\underline{00-0001-6466-} \\
\underline{2802}\end{array}$ \\
\hline $\begin{array}{l}\text { Jhonny Alberto } \\
\text { Botero Guzmán }\end{array}$ & $\begin{array}{l}\text { https://scienti.minciencias.gov.co/cvlac/visualizad } \\
\underline{\text { or/generarCurriculoCv.do?cod } \mathrm{rh}=0001671985}\end{array}$ & $\begin{array}{l}\text { https://scholar.google.com/citations?user=9a } \\
\text { leGpoAAAAJ\&hl=es\&authuser=1 }\end{array}$ & $\begin{array}{l}\underline{\text { https://orcid.org/00 }} \\
\underline{\underline{00-0002-0321-}}\end{array}$ \\
\hline $\begin{array}{l}\text { Claudia Patricia } \\
\text { Tarquino Acosta }\end{array}$ & $\begin{array}{l}\text { https://scienti.minciencias.gov.co/cvlac/visualizad } \\
\underline{\text { or/generarCurriculoCv.do?cod } r h=0000110061}\end{array}$ & $\begin{array}{l}\text { https://scholar.google.es/citations?user=PBf } \\
\text { Et MAAAAJ\&hl=es\&oi=ao }\end{array}$ & $\begin{array}{l}\underline{\text { https://orcid.org/00 }} \\
\underline{00-0003-4811-} \\
\underline{0359}\end{array}$ \\
\hline $\begin{array}{l}\text { Jhon Fernando } \\
\text { Soto Mancera }\end{array}$ & $\begin{array}{l}\text { https://scienti.minciencias.gov.co/cvlac/visualizad } \\
\underline{\text { or/generarCurriculoCv.do?cod } \mathrm{rh}=0000110140}\end{array}$ & $\begin{array}{l}\text { https://scholar.google.es/citations?user=mV } \\
\underline{\text { ckedMAAAAJ\&hl=es\&authuser }=2}\end{array}$ & $\begin{array}{l}\underline{\text { https://orcid.org/00 }} \\
\underline{\underline{00-0003-4724-}}\end{array}$ \\
\hline $\begin{array}{l}\text { William Peñaranda } \\
\text { Zárate }\end{array}$ & $\begin{array}{l}\text { https://scienti.minciencias.gov.co/cvlac/visualizad } \\
\text { or/generarCurriculoCv.do?cod rh=0001222902 }\end{array}$ & $\begin{array}{l}\text { https://scholar.google.es/citations?user=FN } \\
\underline{\text { GSBu4AAAAJ\&hl=es\&oi=ao }}\end{array}$ & $\begin{array}{l}\text { https://orcid.org/00 } \\
\underline{00-0002-4431-} \\
\underline{9078}\end{array}$ \\
\hline
\end{tabular}




\section{Memorias Reflexiones Pedagógicas en los contextos educativos desde las competencias ciudadanas en la sociedad}

La Red Paz Maestros se propone como un escenario académico de articulación entre estudiantes, docentes y personas inquietas por el análisis de los procesos educativos en los cuales, todos nos vemos implicados a través de la práctica pedagógica por las dinámicas entre los procesos de la enseñanza y aprendizaje. ${ }^{2}$

Por lo anterior se planteó un escenario que permitiera la reflexión pedagógica sobre las competencias ciudadanas en los contextos educativos durante el tiempo de confinamiento a causa de la pandemia Covid-19, por lo cual se conto con la participación del Dr. Enrique Chaux quien compartió la conferencia titulada "Competencias ciudadanas para la convivencia pacífica"

El evento fue desarrollado el día 16 de Julio de 2020 de 04:00 pm a 06:00 pm por medio de la plataforma Zoom de la Universidad Santo Tomás con el apoyo del coordinador de TIC de la Sede Villavicencio, el Ing. Jorge Ojeda, contando con intervención del Secretario de Educación Municipal, el Lic Álvaro Hernández Mora, la participación del grupo de investigación Nakota y la asistencia de docentes de distintas instituciones educativas públicas y privadas de la región.

2 Tomado de: Jhonny Alberto Botero Guzmán, J. A., Tarquino Acosta, C. P., Hurtado Hurtado, C. Y., Soto Mancera, J. F. y Contreras Castro, D. A. (2020). Plan estratégico de la red académica y de investigación. Red de Paz Maestros Colombia. Repositorio Universidad Santo Tomás. 


\section{Intervención de Mg. Héctor Rafael Castellanos Líder de Grupo Nakota}

Nakota es un grupo de investigación que centra su búsqueda en las problemáticas del contexto de origen y responde a las necesidades de las comunidades desde objetos de estudio como víctimas del conflicto armado colombiano, las iniciativas de emprendimiento de la sociedad y los aportes de la filosofía y la pedagogía para formación integral de la niñez y de la juventud. De esta manera se proyecta en concordancia con la misión humanista de la USTA ${ }^{1}$

1 Tomado de: Ramírez Martínez, J. E. y Suárez Sandoval, M. C. (eds.). (2020). La investigación en USTA, Sede Villavicencio:

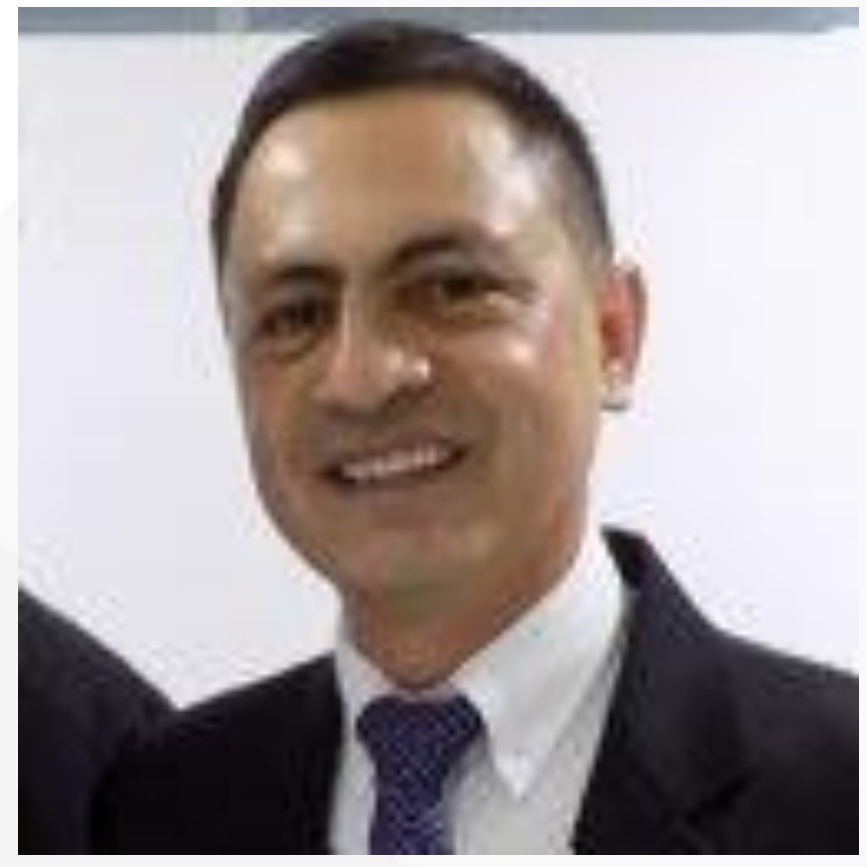
capacidades CTel desde los grupos de investigación. Ediciones USTA 


\section{Intervención del Secretario de Educación Municipal Lic Álvaro Hernández Mora}

\section{6}

Este es un escenario para el debate y para pensar la formación de ciudadanos que demandan la contemporaneidad, en cara a este mundo globalizado en el que nos encontramos frente al Covid- 19 lo cual nos demanda una reflexión entorno a qué es ser humano y qué es la humanidad (...) Hablar de ciudadanía implica pensar en la formación de ciudadanos desde la triada: autonomía responsabilidad y libertad. Sin embargo la ciudadanía debe pasar por la dignidad, la dignidad una en términos de paz que implica justicia económica y social.

\section{5}

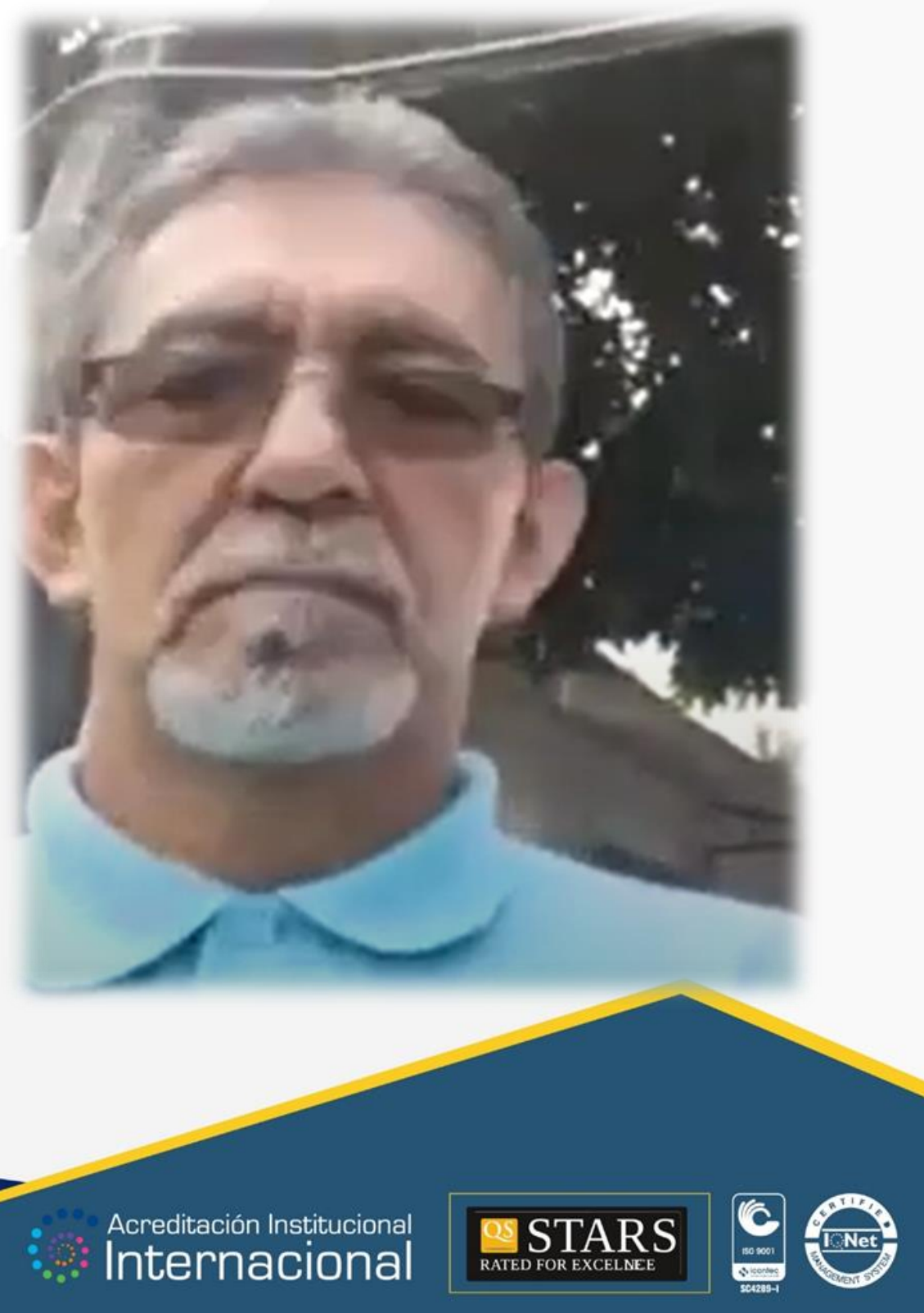


Espacio de intervención de la experiencia de instituciones educativas rurales durante la pandemia a través de los flash movies por parte de la docente Claudia Yasmin Hurtado de la Institución educativa Apiay.
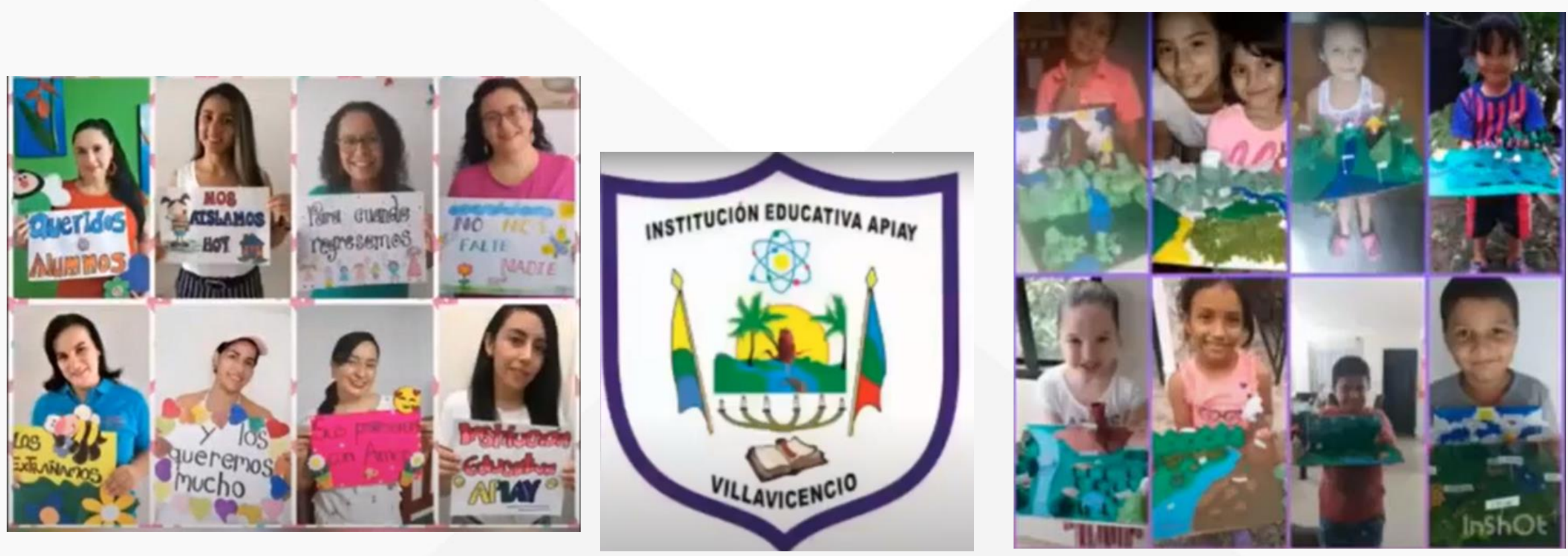

隊: UNIVERSIDAD SANTOTOMAS PRIMER CLAUSTRO UNIVERSITARIO DE COLOMBIA

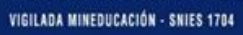

\section{PATSARS}

둥우 


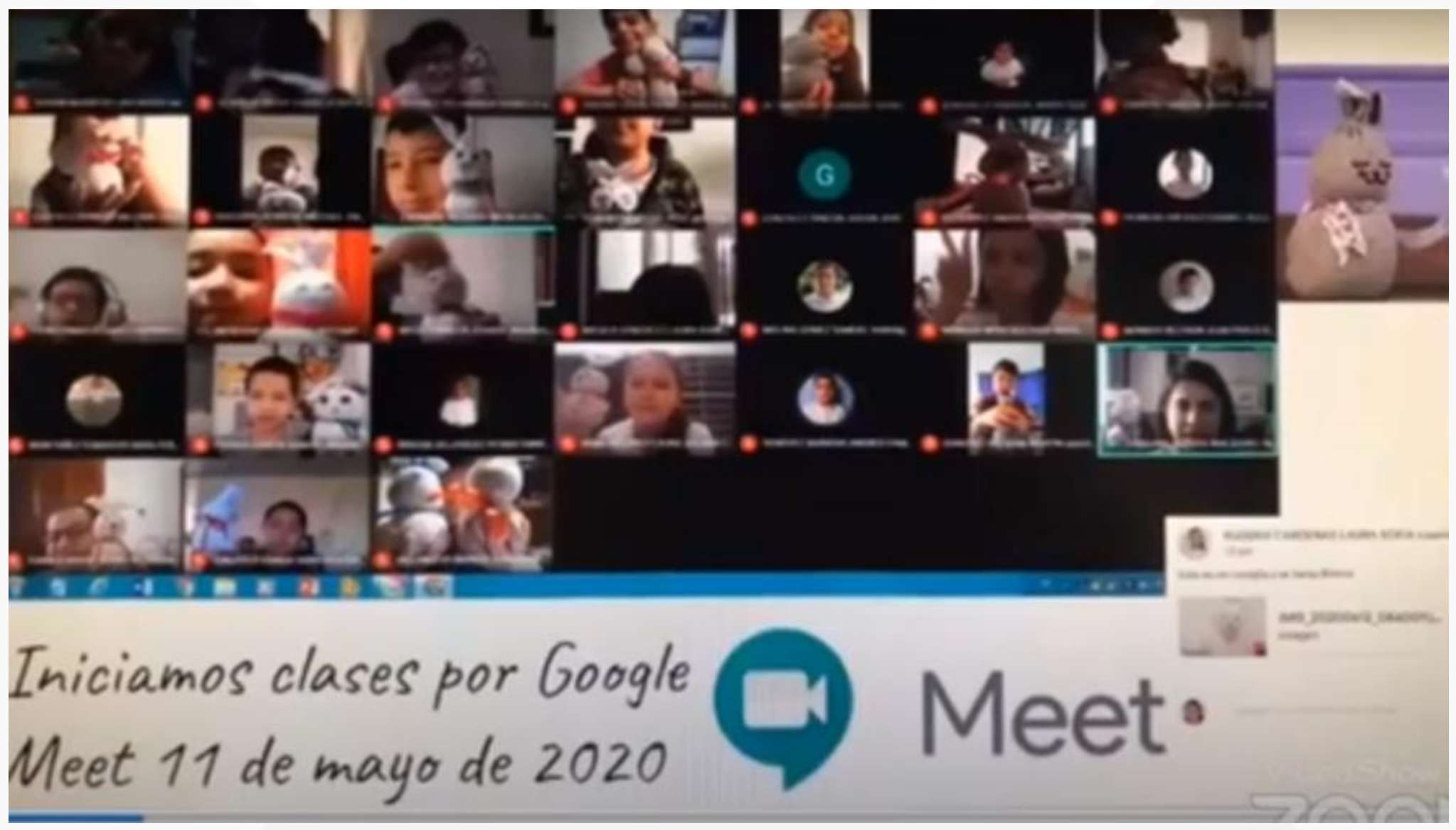

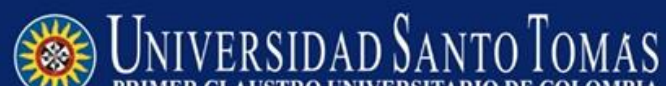

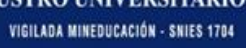

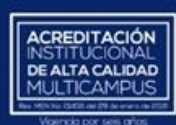
$\therefore$ Acreditación Institucional RATED FOR EXCELNEE 


\section{Reflexión pedagógica en el contexto urbano experiencia en el Gimnasio Campestre la Fontana por el Lic. Daniel Alejandro Contreras}

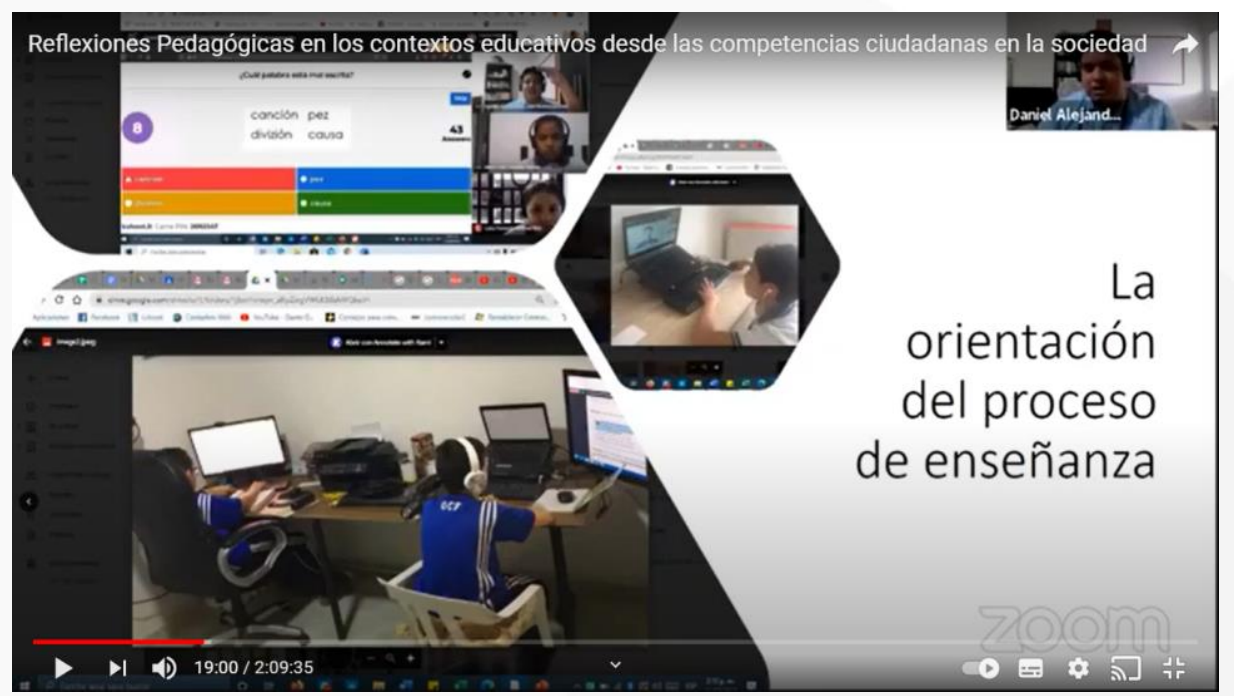

El Gimnasio Campestre la Fontana ante las circunstancias que hemos vivimos ha visto oportunidades para alcanzar nuevos logros que demuestran el compromiso por orientar una educación asistida por las tecnologías en las cuales se implementaron espacios educativos desde casa en los que estudiantes en compañía de docentes y padres de familia se apoyaban en el proceso de enseñanza y aprendizaje con ayuda de plataformas como Classromm, Meet, Kahoot, entre otras, que permitieron no perder experiencias dentro de la formación académica, musical, artística y deportiva propias de la institución.

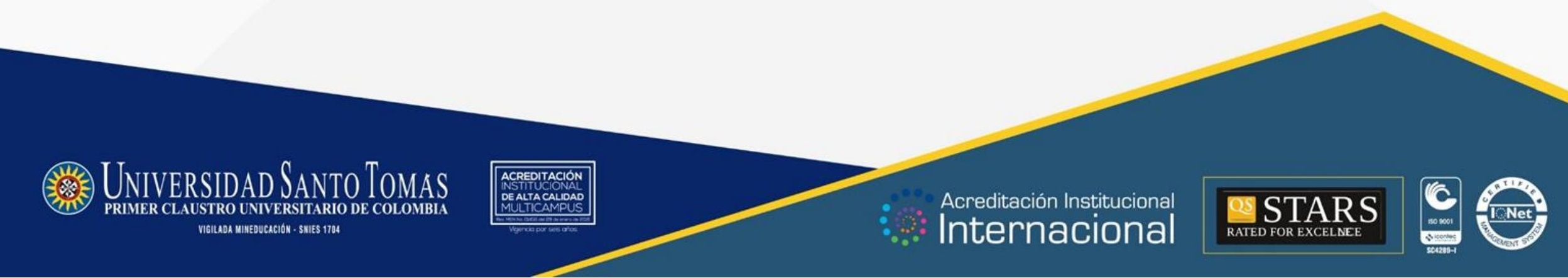




\section{Conferencia Competencias ciudadanas para la convivencia pacífica Dr. Enrique Chaux}

El Doctor Chaux realizó una descripción de los contenidos de su programa pedagógico "Aulas en Paz" en donde contó historias de personajes que han sido victimas de acoso escolar con el propósito de demostrar una estrategia pedagógica con la cual se puede fomentar espacios que generen en los estudiantes diversas vivencias y sentimientos como la empatía que permiten una reflexión frente a los actos violentos vivenciados en el contexto escolar.

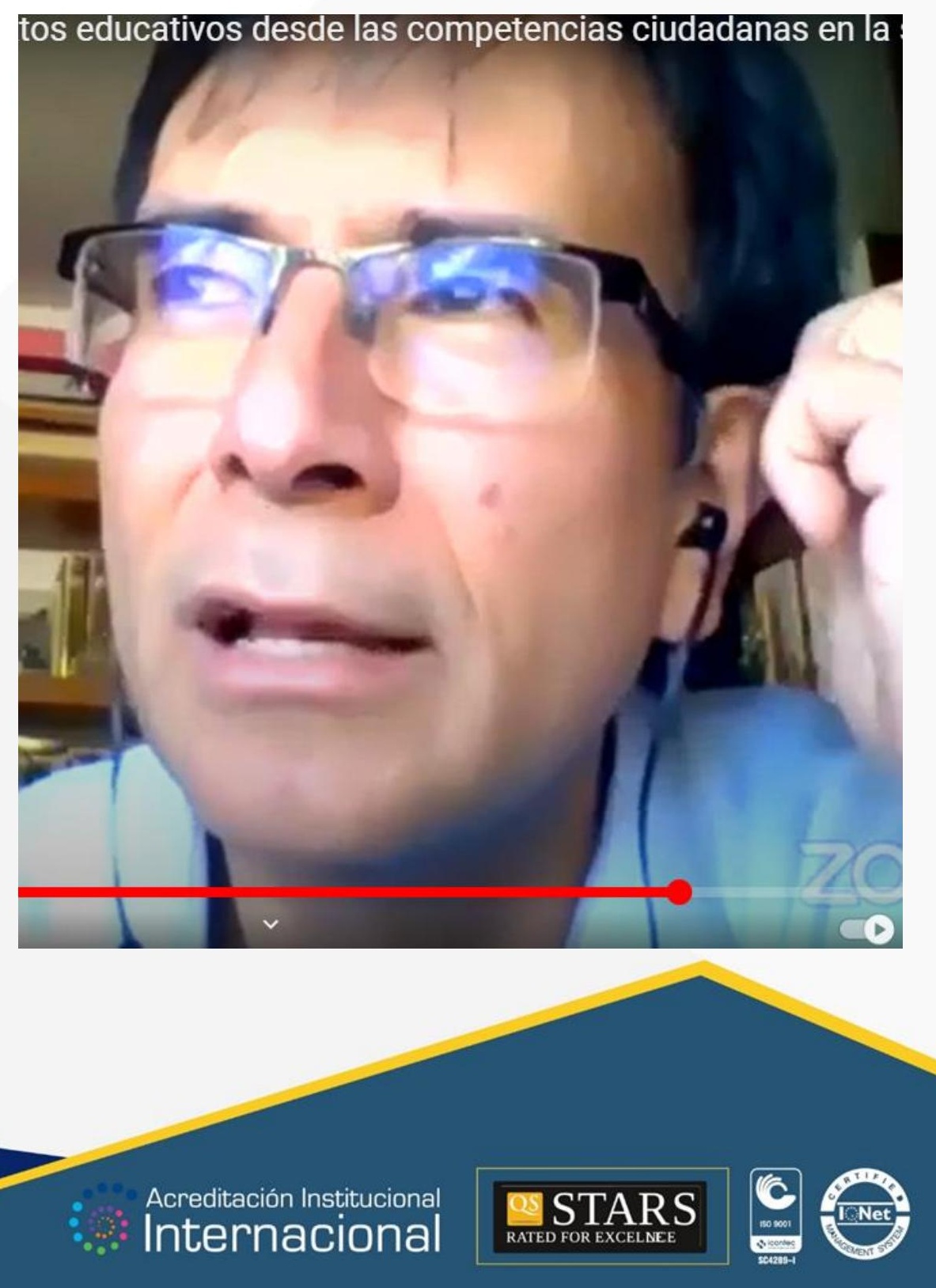


Nosotros no leemos el libro de manera tradicional que al final viene una moraleja, lo que hacemos es que vamos leyendo haciendo preguntas en momentos cruciales que busca desarrollar las competencias, como: ¿Has sentido algo parecido a Oliver? ¿Conoces ha alguien que ha vivido la misma situación con Oliver?, como en este caso, se trabaja la empatía. 
La capacidad de pensar en síntesis, en alternativas en donde ambos podemos estar de acuerdo, que sean mejores que las posiciones iniciales, es una de las habilidades cruciales para la solución de conflictos 59

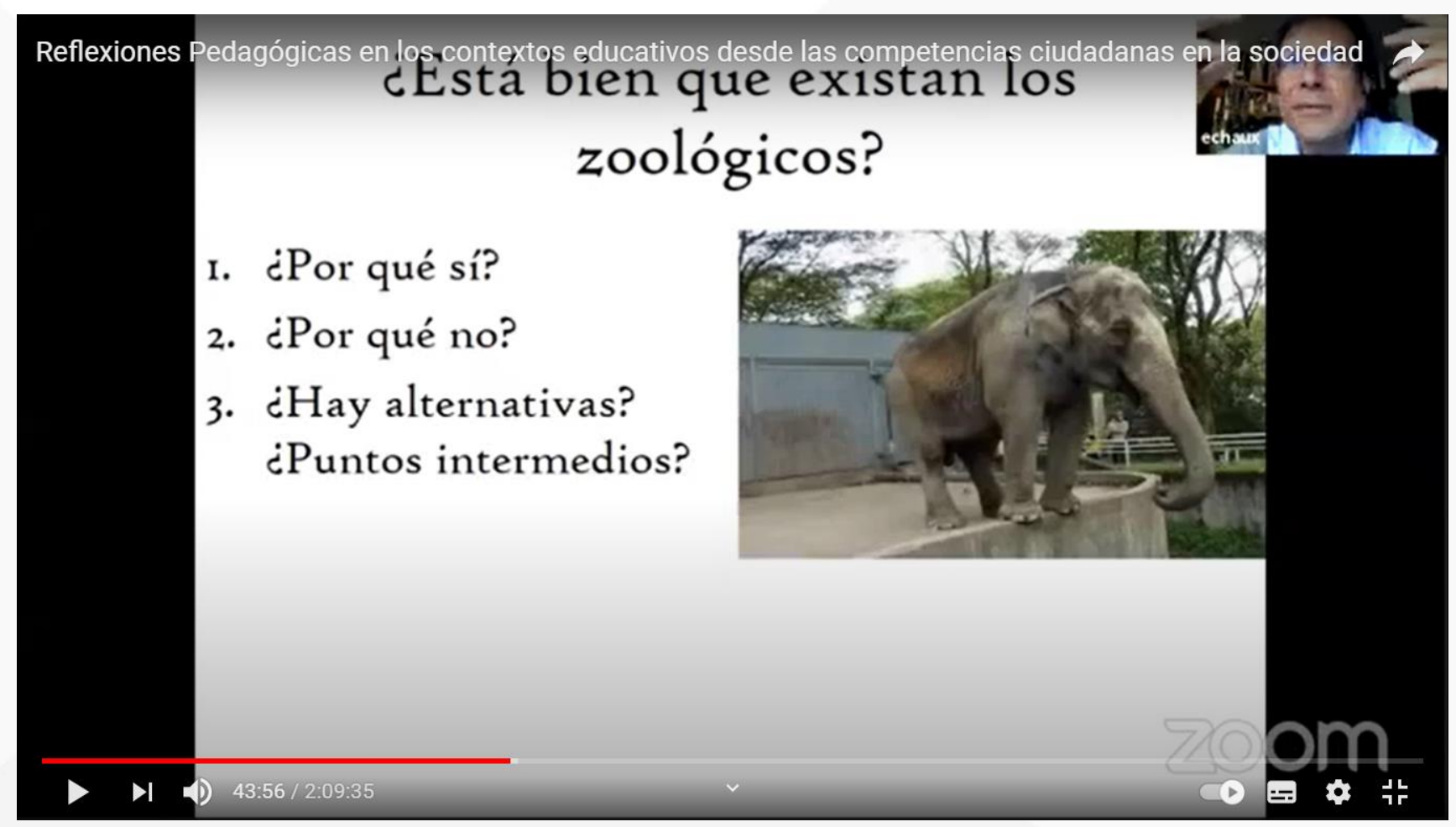




\section{6}

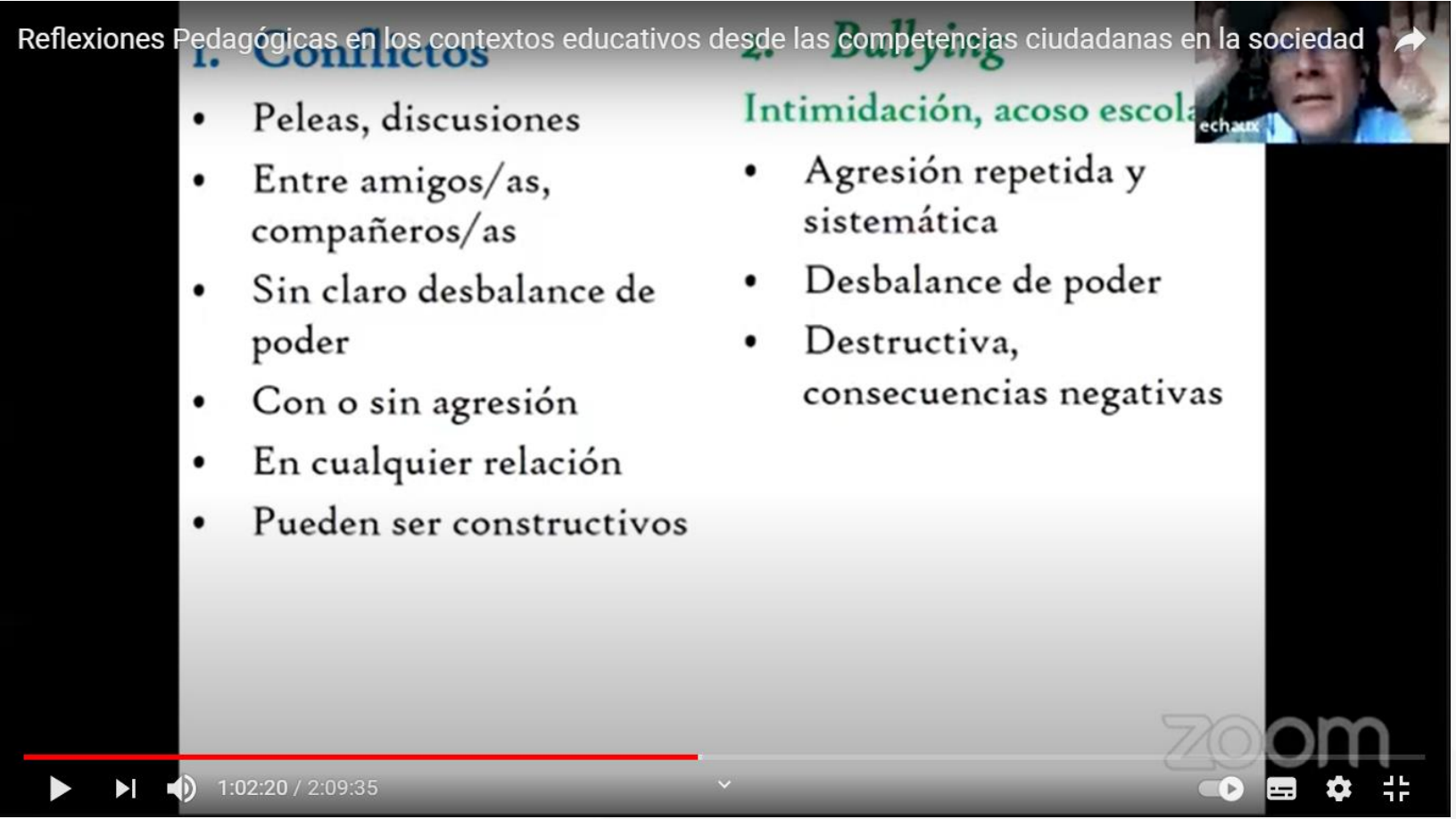

Estas dos son situaciones que son típicas en el contexto de la vida de los niños, niñas y jóvenes, que son muy frecuentes. En realidad estás situaciones reflejan dos dinámicas bastantes distintas que pueden generar bastante violencia.

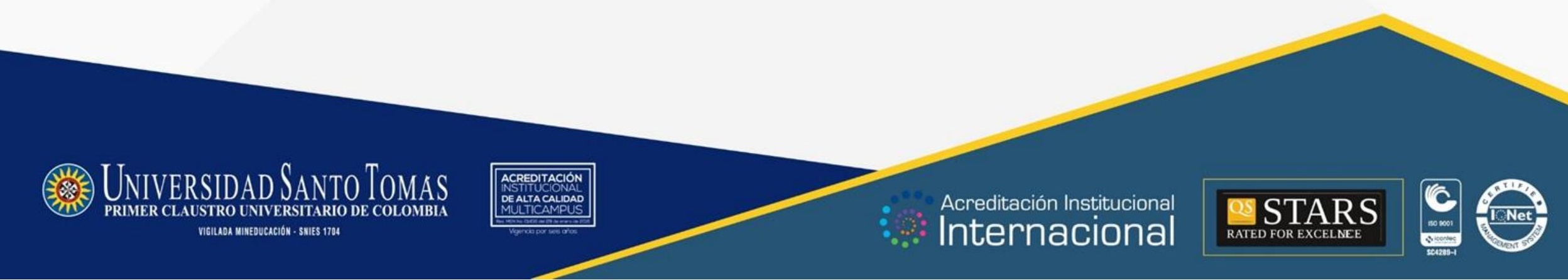


Los colegios en Colombia según datos del ICFES que tienen más Bullying, son aquellos en los que existe menos empatía, teniendo ésta una relación inversa. Sí hay conexión con los demás, es menos probable participar en el maltrato a otra.

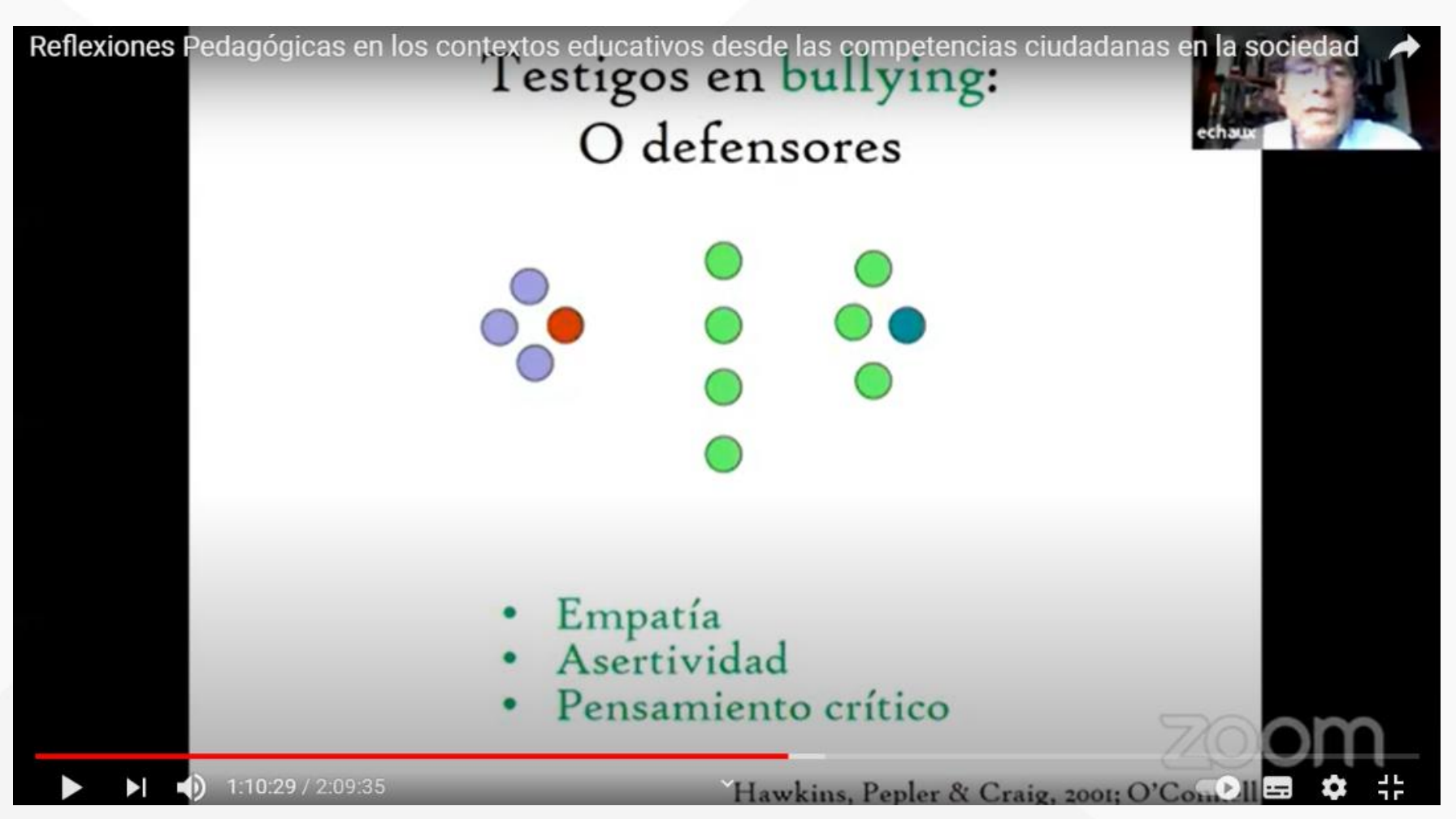




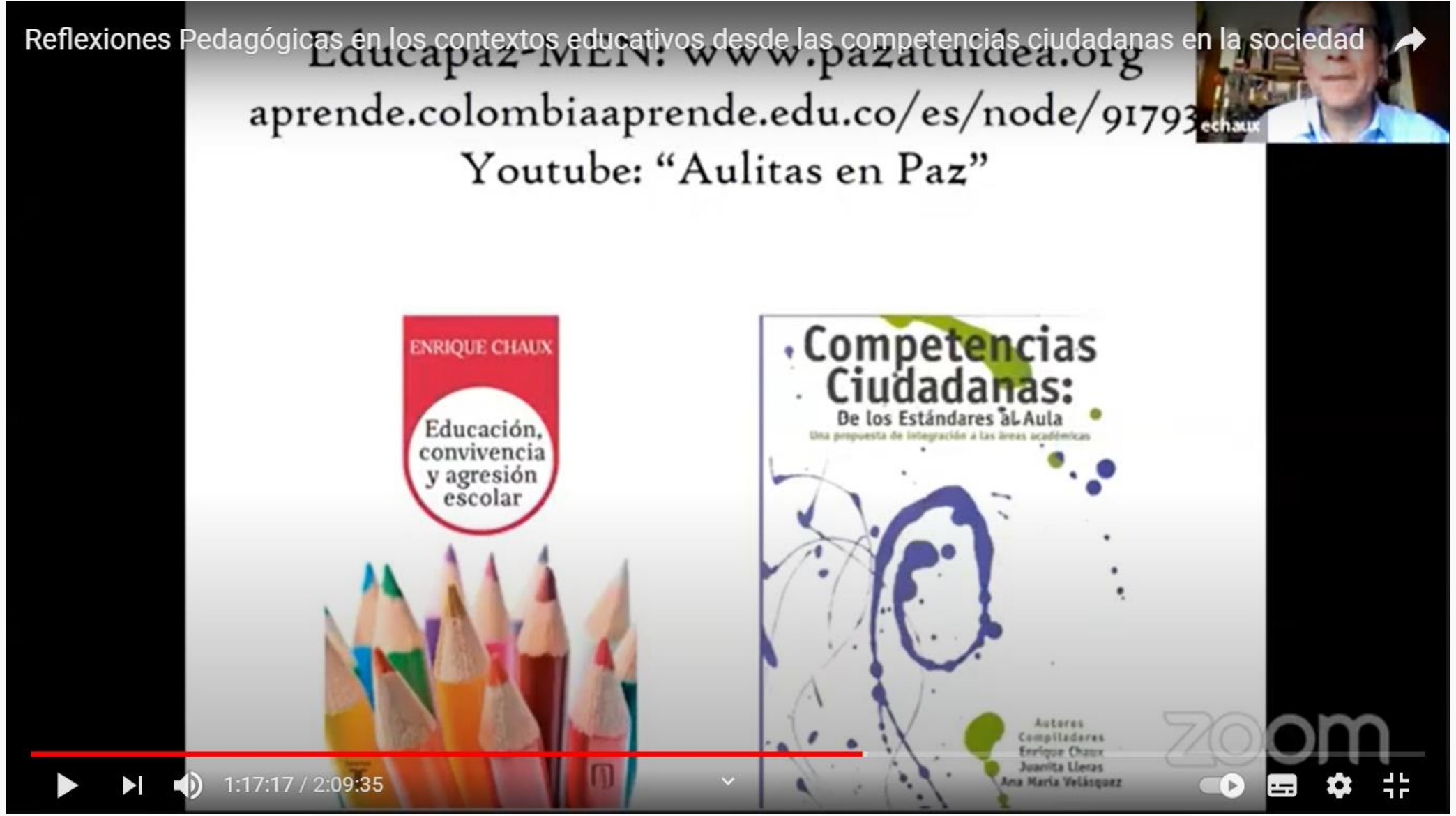

Los invitamos a ver la conferencia completa disponible en: https://www.youtube.com/watch?v=Ir2xm2LkQiU\&feature=youtu.be 


\section{LOGROS}

El conversatorio contó con la participación de 102 profesores de diferentes instituciones educativas de la región, quienes evaluaron el evento de la siguiente manera:

¿El contenido del tema expuesto genera ímpacto en el contexto educativo actual?

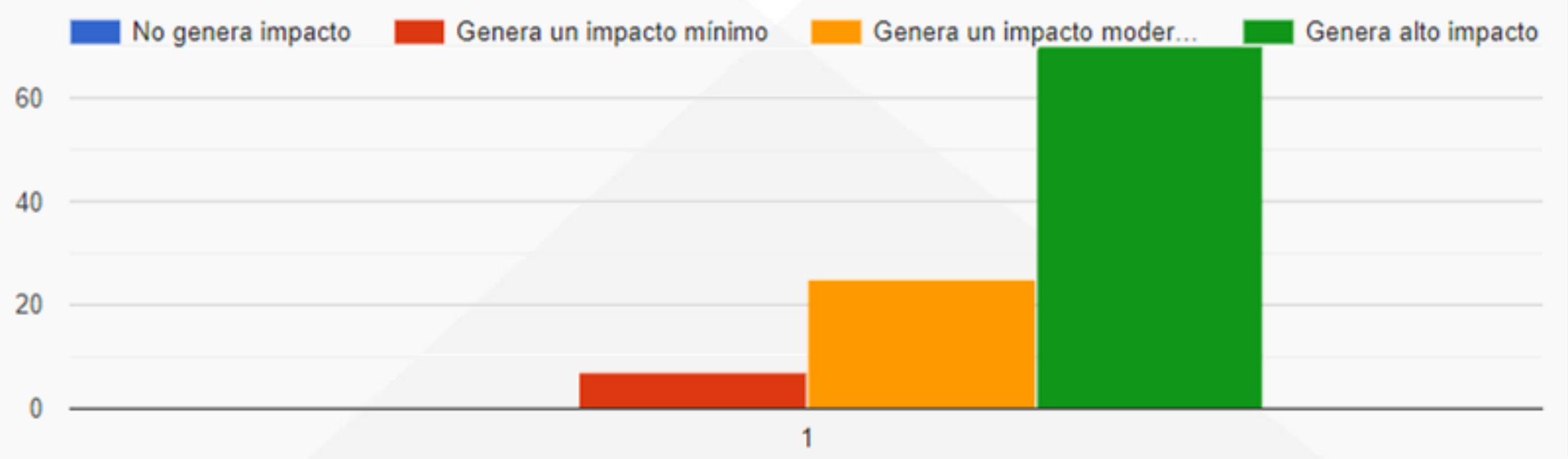


¿El evento cumplio̊ con sus expectativas?

102 respuestas

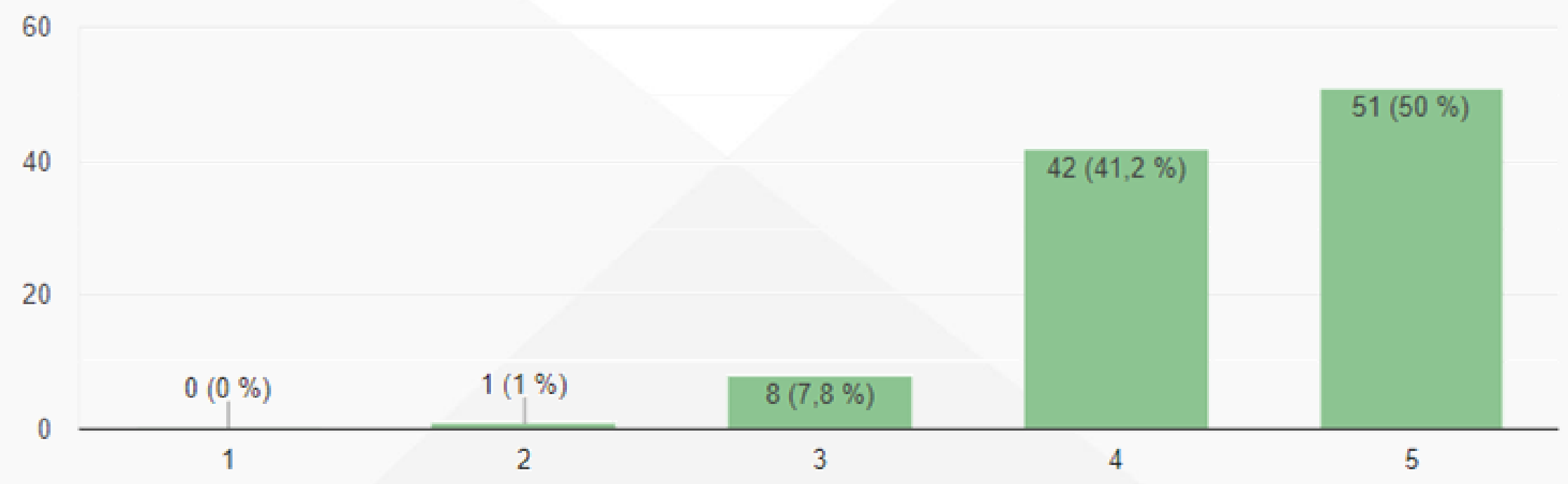




\section{REFERENCIAS}

Jhonny Alberto Botero Guzmán, J. A., Tarquino Acosta, C. P., Hurtado Hurtado, C. Y., Soto Mancera, J. F. y Contreras Castro, D. A. (2020). Plan estratégico de la red académica y de investigación. Red de Paz Maestros Colombia. Repositorio Universidad Santo Tomás

Ramírez Martínez, J. E. y Suárez Sandoval, M. C. (eds.). (2020). La investigación en USTA, Sede Villavicencio: capacidades CTel desde los grupos de investigación. Ediciones USTA

Castellanos, H., Barbosa, J., Araujo, L., Peñaranda, W., Neira, D., Contreras, D., Soto, J., Guerrero, B., Palomino, C., Palomino, J., Zuluaga, J., Botero, J., Rodríguez, L., Galvis, V., Tiuso, A., Vega, J., Florián, H., Triviño, L., González, H., Alzate, L., Santacruz, A., Chávez, O., Rubio, D. (2020). Presentación signo distintivo Grupo de Investigación Nakota. Universidad Santo Tomás. Villavicencio

Vega, J., Araujo, L., González, H., Peñaranda, W., Botero, J., Contreras, D., Soto, J., Ulloa, C., Tarquino, C. Presentación Signo Distintivo de la Red Paz Maestros Colombia. Universidad Santo Tomás. Villavicencio 